\title{
A REMARK ON THE RESTRICTION MAP IN FIELD FORMATION
}

\author{
HIRONORI ONISHI
}

\begin{abstract}
In this note we point out that in a field formation $\left(G,\left\{G_{F}\right\}, A\right)$, if $h_{2}(K / F)=[K: F]^{c}$ for every normal layer $K / F$ with a fixed integer $c \geq 0$, then for every tower $F \subset E \subset K$ with $K / F$ normal, the restriction map $H^{2}(K / F) \rightarrow H^{2}(K / E)$ is surjective, and give an example with $c=2$.
\end{abstract}

Let $\left(G,\left\{G_{F}\right\}, A\right)$ be a field formation. (For the notations and basic facts, see [1, Chapter 14, pp. 197-209].) Then for every tower $F \subset K \subset L$ with $K / F$ and $L / F$ normal, the sequence

$$
0 \rightarrow H^{2}(K / F) \stackrel{\text { inf }}{\longrightarrow} H^{2}(L / F) \stackrel{\text { res }}{\longrightarrow} H^{2}(L / K)
$$

is exact. We shall prove that if $h_{2}(K / F)=[K: F]^{c}$ for every normal layer $K / F$ with a fixed integer $c \geq 0$, then for every tower $F \subset E \subset K$ with $K / F$ normal, res: $H^{2}(K / F) \rightarrow H^{2}(K / E)$ is surjective.

If $F \subset K \subset L$ with $K / F$ and $L / F$ normal, then the exact sequence (1) gives that the order of the image res $H^{2}(L / F)$ is equal to $[L: F]^{c} /[K: F]^{c}=[L: K]^{c}$ $=h_{2}(L / K)$. Thus res: $H^{2}(L / F) \rightarrow H^{2}(L / K)$ is surjective in this case.

Let $F \subset E \subset K$ with $K / F$ normal. For each prime $p$, the restriction map takes the $p$-primary component $H^{2}(K / F)_{p}$ of $H^{2}(K / F)$ into the $p$-primary component $H^{2}(K / E)_{p}$ of $H^{2}(K / E)$. Thus it is enough to show that res: $H^{2}(K / F)_{p} \rightarrow H^{2}(K / E)_{p}$ is surjective for every $p$.

Let $G_{K / E_{0}}$ be a $p$-Sylow subgroup of $G_{K / E}$. Then by a Sylow theorem, there exists a chain

$$
G_{K / E_{0}}=G_{K / F_{r}} \subset G_{K / F_{r-1}} \subset \cdots \subset G_{K / F_{0}}
$$

of $p$-subgroups of $G_{K / F}$ such that $G_{K / F_{i}}$ is normal in $G_{K / F_{i-1}}$ for each $i=1, \ldots$, $r$ and $G_{K / F_{0}}$ is a $p$-Sylow subgroup of $G_{K / F}$. We know that the restriction map takes $H^{2}(K / E)_{p}$ injectively into $H^{2}\left(K / E_{0}\right)$. In our case,

$$
\text { res: } H^{2}(K / E)_{p} \rightarrow H^{2}\left(K / E_{0}\right)
$$

is bijective because both $H^{2}(K / E)_{p}$ and $H^{2}\left(K / E_{0}\right)$ have the same order $\left[K: E_{0}\right]^{c}$. Likewise

$$
\text { res: } H^{2}(K / F)_{p} \rightarrow H^{2}\left(K / F_{0}\right)
$$

is bijective. Thus it is sufficient to show that

Received by the editors January 29, 1975 and, in revised form, June 2, 1975.

AMS (MOS) subject classifications (1970). Primary 12A60.

Key words and phrases. Field formation. 


$$
\text { res: } H^{2}\left(K / F_{0}\right) \rightarrow H^{2}\left(K / E_{0}\right)
$$

is surjective. This map factors as

$$
H^{2}\left(K / F_{0}\right) \stackrel{\text { res }}{\longrightarrow} H^{2}\left(K / F_{1}\right) \stackrel{\text { res }}{\longrightarrow} \cdots \stackrel{\text { res }}{\longrightarrow} H^{2}\left(K / F_{r}\right) .
$$

Since $K / F_{i}$ and $F_{i} / F_{i-1}$ are normal, each factor

$$
\text { res: } H^{2}\left(K / F_{i-1}\right) \rightarrow H^{2}\left(K / F_{i}\right)
$$

is surjective. Thus the composite (2) of these is surjective. This completes the proof of the remark.

We now offer an example of field formation in which $h_{2}(K / F)=[K: F]^{2}$ for every normal layer $K / F$. Let $p$ be a rational prime and $\mathbf{Q}_{p}$ be the rational $p$-adic number field. Let $P=\mathbf{Q}_{p}\{t\}$, the field of formal power series in $t$ over $\mathbf{Q}_{p}$. Let $\Omega$ be the splitting field of the polynomials $X^{n}-t$ over $P$ for all integers $n>0$ not divisible by $p$. Given a finite extension $F$ of $P$ in $\Omega$, let $G_{F}$ be the Galois group of $\Omega / F$. Then $\left(G,\left\{G_{F}\right\}, \Omega^{\times}\right)$, where $G=G_{P}$ and $\Omega^{\times}$is the multiplicative group of $\Omega$, is a field formation. We claim that $h_{2}(K / F)$ $=[K: F]^{2}$ for every normal layer $K / F$ in $\Omega / P$.

The ground field $P$ is complete under the nonarchimedian valuation | given by $|x|=e^{-r}$ if

$$
x=\sum_{k \geq r} a_{k} t^{k}, \quad a_{k} \in \mathbf{Q}_{p}, a_{r} \neq 0,
$$

and the valuation is extended to $\Omega$. Given a field $K$ in the formation, let $\theta_{K}$, $M_{K}$ and $U_{K}$ be the valuation ring, its maximal ideal and the group of units in $\vartheta_{K}$, respectively. Let $\bar{K}=\vartheta_{K} / M_{K}$ and $U_{K}^{1}=1+M_{K}$. The residue field $\bar{K}$ is an abelian extension of $\mathbf{Q}_{p}$.

Since every normal layer in our formation is solvable, by induction we see that it is sufficient to prove the equality $h_{2}(K / F)=[K: F]^{2}$ for every cyclic layer $K / F$ of prime degree. For this it is sufficient to establish the equality in the following two cases: (a) when $K / F$ is unramified and (b) when $K / F$ is totally ramified.

For any normal layer $K / F$, we have $H^{q}\left(G_{K / F}, U_{K}^{1}\right)=0$ for all $q$ because $x \mapsto x^{n}$ is an automorphism of $U_{K}^{1}$. Thus the exact sequence

$$
0 \rightarrow U_{K}^{1} \rightarrow U_{K} \rightarrow \bar{K}^{\times} \rightarrow 0
$$

gives that

$$
H^{q}\left(G_{K / F}, U_{K}\right)=H^{q}\left(G_{K / F}, \bar{K}^{\times}\right)
$$

for all $q$. While the exact sequence

$$
0 \rightarrow U_{K} \rightarrow K^{\times} \stackrel{\nu_{K}}{\longrightarrow} \mathbf{Z} \rightarrow 0,
$$

where $\nu_{K}$ is the exponential valuation on $K$, gives the long exact sequence

$$
0 \rightarrow H^{2}\left(G_{K / F}, U_{K}\right) \rightarrow H^{2}(K / F) \rightarrow H^{2}\left(G_{K / F}, \mathbf{Z}\right) \stackrel{\delta}{\rightarrow} \cdots
$$


Note that

$$
H^{2}\left(G_{K / F}, \mathbf{Z}\right)=H^{1}\left(G_{K / F}, \mathbf{Q} / \mathbf{Z}\right)=G_{K / F}^{*},
$$

the character group of $G_{K / F}$.

CASE (a). $K / F$ is unramified. Since $G_{K / F}=G_{K / \bar{F}}$ (3) gives that

$$
H^{q}\left(G_{K / F}, U_{K}\right)=H^{q}(\bar{K} / \bar{F}) .
$$

Since the exact sequence (4) splits in this case, we get the exact sequence

$$
0 \rightarrow H^{2}(\bar{K} / \bar{F}) \rightarrow H^{2}(K / F) \rightarrow G_{K / F}^{*} \rightarrow 0 .
$$

Since $K / F$ is abelian, $\left|G_{K / F}^{*}\right|=[K: F]$. While by the local class field theory, $h_{2}(\bar{K} / \bar{F})=[\bar{K}: \bar{F}]=[K: F]$. Thus we get that $h_{2}(K / F)=[K: F]^{2}$.

CASE (b). $K / F$ is totally ramified. Then $\bar{K}=\bar{F}$ and this field contains a primitive $n$th root of unity, where $n=[K: F]$, and $K / F$ is cyclic. Thus

$$
H^{3}\left(G_{K / F}, \bar{K}^{\times}\right)=H^{1}\left(G_{K / F}, \bar{F}^{\times}\right)
$$

is of order $n$. Since $H^{3}(K / F)=H^{1}(K / F)=0$ and $\left|G_{K / F}^{*}\right|=n$,

$$
\delta: G_{K / F}^{*} \rightarrow H^{3}\left(G_{K / F}, \bar{K}\right)
$$

is an isomorphism. Thus (5) gives that

$$
H^{2}(K / F)=H^{2}\left(G_{K / F}, \bar{K}^{\times}\right)=H^{0}\left(G_{K / F}, \bar{F}^{\times}\right)=\bar{F}^{\times} / \bar{F}^{\times n} .
$$

But since $(n, p)=1,\left[\bar{F}^{\times}: \bar{F}^{\times n}\right]=n^{2}$. Thus $h_{2}(K / F)=[K: F]^{2}$.

\section{REFERENCE}

1. E. Artin and J. Tate, Class field theory, Benjamin, New York and Amsterdam, 1968. MR 36 \#6383.

Department of Mathematics, City College (CUNY), New York, New York 10031 